\title{
A short, robust brain activation control task optimised for pharmacological fMRI studies
}

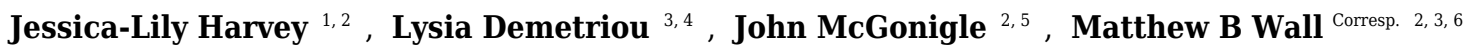 \\ ${ }^{1}$ School of Psychology and Neuroscience, University of St. Andrews, St Andrews, United Kingdom \\ 2 Division of Brain Sciences, Imperial College London, London, United Kingdom \\ 3 Invicro Ltd., London, United Kingdom \\ 4 Department of Medicine, Imperial College London, London, United Kingdom \\ 5 Perspectum Diagnostics, Oxford, United Kingdom \\ Clinical Psychopharmacology Unit, University College London, University of London, London, United Kingdom \\ Corresponding Author: Matthew B Wall \\ Email address: matthew.wall@imperial.ac.uk
}

Background. Functional magnetic resonance imaging (fMRI) is a popular method for examining pharmacological effects on the brain; however the BOLD response is dependent on intact neurovascular coupling, and potentially modulated by a number of physiological factors. Pharmacological fMRI is therefore vulnerable to confounding effects of pharmacological probes on general physiology or neurovascular coupling. Controlling for such non-specific effects in pharmacological fMRI studies is therefore an important consideration, and there is an additional need for well-validated fMRI task paradigms that could be used to control for such effects, or for general testing purposes.

Methods. We have developed two variants of a standardized control task that are short (5 minutes duration) simple (for both the subject and experimenter), widely applicable, and yield a number of readouts in a spatially diverse set of brain networks. The tasks consist of four functionally discrete threesecond trial types (plus additional null trials) and contain visual, auditory, motor and cognitive (eyemovements, and working memory tasks in the two task variants) stimuli. Performance of the tasks was assessed in a group of 15 subjects scanned on two separate occasions, with test-retest reliability explicitly assessed using intra-class correlation coefficients.

Results. Both tasks produced robust patterns of brain activation in the expected brain regions, and region of interest-derived reliability coefficients for the tasks were generally high, with four out of eight task conditions rated as 'excellent' or 'good', and only one out of eight rated as 'poor'. Median values in the voxel-wise reliability measures were also $>0.7$ for all task conditions, and therefore classed as 'excellent' or 'good'. The spatial concordance between the most highly activated voxels and those with the highest reliability coefficients was greater for the sensory (auditory, visual) conditions than the other (motor, cognitive) conditions.

Discussion. Either of the two task variants would be suitable for use as a control task in future pharmacological fMRI studies or for any other investigation where a short, reliable, basic task paradigm is required. Stimulus code is available online for re-use by the scientific community. 


\title{
1 A short, robust brain activation control task optimised for 2 pharmacological fMRI studies.
}

3 Jessica-Lily Harvey ${ }^{1,2}$, Lysia Demetriou ${ }^{3,4}$, John McGonigle ${ }^{2,5}$, and Matthew B Wall ${ }^{2,3,6}$.

$4 \quad{ }^{1}$ University of St. Andrews, School of Psychology and Neuroscience, St Andrews, UK.

$5 \quad 2$ Division of Brain Sciences, Imperial College London, London, UK

6 3Invicro London, Hammersmith Hospital, London, UK

$7 \quad{ }^{4}$ Department of Medicine, Imperial College London, London, UK

$8 \quad 5$ Perspectum Diagnostics, Oxford, UK.

$9{ }^{6}$ Clinical Psychopharmacology Unit, University College London, London, UK.

\author{
Corresponding Author: \\ Matthew Wall \\ Invicro London \\ Burlington Danes Building \\ Hammersmith Hospital \\ Du Cane Road \\ London, W12 0NN \\ United Kingdom \\ matt.wall@invicro.co.uk
}




\section{Abstract}

25 Background. Functional magnetic resonance imaging (fMRI) is a popular method for examining pharmacological effects on the brain; however the BOLD response is dependent on intact neurovascular coupling, and potentially modulated by a number of physiological factors. Pharmacological fMRI is therefore vulnerable to confounding effects of pharmacological probes on general physiology or neurovascular coupling. Controlling for such non-specific effects in pharmacological fMRI studies is therefore an important consideration, and there is an additional need for well-validated fMRI task paradigms that could be used to control for such effects, or for general testing purposes.

33 Methods. We have developed two variants of a standardized control task that are short (5 minutes duration) simple (for both the subject and experimenter), widely applicable, and yield a number of readouts in a spatially diverse set of brain networks. The tasks consist of four functionally discrete three-second trial types (plus additional null trials) and contain visual, auditory, motor and cognitive (eye-movements, and working memory tasks in the two task variants) stimuli. Performance of the tasks was assessed in a group of 15 subjects scanned on two separate occasions, with test-retest reliability explicitly assessed using intra-class correlation coefficients.

41 Results. Both tasks produced robust patterns of brain activation in the expected brain regions, and region of interest-derived reliability coefficients for the tasks were generally high, with four out of eight task conditions rated as 'excellent' or 'good', and only one out of eight rated as 'poor'. Median values in the voxel-wise reliability measures were also $>0.7$ for all task conditions, and therefore classed as 'excellent' or 'good'. The spatial concordance between the most highly activated voxels and those with the highest reliability coefficients was greater for the sensory (auditory, visual) conditions than the other (motor, cognitive) conditions.

Discussion. Either of the two task variants would be suitable for use as a control task in future pharmacological fMRI studies or for any other investigation where a short, reliable, basic task paradigm is required. Stimulus code is available online for re-use by the scientific community. 


\section{Introduction}

52

53 Functional Magnetic Resonance Imaging (fMRI) is currently one of the major standard methods

54 in cognitive neuroscience research. FMRI provides reasonably high spatial and temporal

55 resolution data, is flexible enough to accommodate a wide variety of experimental designs, and exposure to magnetic fields presents no danger to most subjects (Logothetis, 2008; Soares et al., 2016). FMRI can also be used as an index of pharmacological effects; drugs or hormones can be administered before or during a scanning session, and the results compared with a baseline or placebo session (e.g. Carhart-Harris et al., 2014; Comninos et al., 2017; Kaelen et al., 2016; Upadhyay et al., 2011). Pharmacological-fMRI studies may be used in the drug discovery process (Wise \& Tracey, 2006; Matthews, Rabiner \& Gunn, 2011; Carmichael et al., 2018), in the characterization of the effects of commonly-prescribed drugs (Maron et al., 2016), or in the exploration of disorders such as addiction (Quelch et al., 2017).

Conducting pharmacological-fMRI investigations presents many of the same challenges as standard fMRI, but also has some unique issues. One fundamental concern is related to the fact that (most commonly) fMRI studies use the BOLD (Blood-Oxygen-Level-Dependent) signal as the primary end-point. This is a contrast produced by local changes in the ratio of oxygenated and de-oxygenated hemoglobin (Buxton, Wong \& Frank, 1998; Friston et al., 2000), and is usually regarded as a proxy measure of neural activity. However, the relationship between neural activity and this vascular response (neurovascular coupling) is complex and relies on a number of cellular and metabolic processes (Logothetis et al., 2001). Use of a pharmacological agent combined with $\mathrm{fMRI}$ means that any differences observed in the BOLD response may be a combination of direct neural effects of the drug (usually the effects of interest), and indirect effects of the drug (e.g. on neurovascular coupling, or global, systemic effects on blood-pressure, cerebral blood flow, heart-rate, etc.; usually regarded as confounding effects). One example is caffeine, which has direct neural effects on adenosine $A_{1}$ and $A_{2 a}$ receptors, but is also a

77 powerful cerebral vasoconstrictor (Diukova et al., 2012). Separating the neural and vascular 78 effects of even such a well-studied drug as caffeine is therefore a considerable challenge. For detailed reviews of these issues see Bourke and Wall (2015), and Iannetti and Wise (2007). 
81 work by Stewart et al. ( 2014) has shown that Arterial Spin Labelling (ASL) MRI may be a

82 superior technique to assess some pharmacological effects, largely because of its stability across

83 longer periods of time. Other techniques such as magnetoencephalography (MEG) show great

84 promise (Baillet, 2017) but have yet to achieve the broad availability and application of BOLD-

85 fMRI. The flexibility of BOLD-fMRI in terms of task paradigms, its 'good-enough' spatial and

86 temporal resolution (Logothetis, 2008), and its broad availability and relative ease of use mean it

87 is still a popular method in pharmacological investigations.

88 While BOLD-fMRI experiments typically incorporate intrinsic control conditions, these may not 89 adequately control for some hypothetical pharmacological effects. If the drug produces a simple 90 amplitude shift in the responses to both (experimental, and control) conditions, the control is still 91 effective and the effect on the experimental > control contrast will be negligible. However, if the 92 drug a) selectively affects one condition, and not the other, or b) produces a non-linear effect on 93 the difference between the conditions, the intrinsic control may become unreliable. Also, many 94 experiments have used a simple baseline (resting) control condition (e.g. Wise et al., 2004). In 95 such cases, amplitude shifts to the baseline as a result of non-neural effects could be very 96 problematic. One method of mitigating this problem is the use of an independent control task 97 paradigm as part of a pharmacological fMRI scanning session (Iannetti \& Wise, 2007). For 98 example, Murphy et al. (2009) used a visual control task in their study of the effect of citalopram 99 on amygdala responses to emotional faces. In this case the lack of effect of the drug on the visual 100 control task suggests that the effects seen in the main task are unlikely to be due to effects on 101 neurovascular coupling, or other global/systemic effects. However, the use of a single (visual) 102 control task, which gives activation in a circumscribed region of the brain (the occipital lobe) is 103 suboptimal as effects on neurovascular coupling may conceivably vary across the brain.

104 Comninos et al. (2017) used a much more elaborate control task (based on Pinel et al., 2007) in 105 their recent study on the sex hormone kisspeptin. This task involved ten trial conditions which 106 gave results in five separate functional domains (visual, auditory, language, motor, and 107 cognitive), and in a much wider spatial distribution across the brain. This task involved relatively 108 complex instructions for the subjects, and also included some culturally-specific language 109 stimuli, which somewhat limits its broad applicability.

110 An ideal task for the control of pharmacological fMRI studies should have the following 
111 characteristics. First, it should be short in duration as it generally has to be included as part of a

112 broader set of functional task paradigms, anatomical scans, and perhaps other MRI measures

113 (resting-state fMRI, perfusion measures, spectroscopy etc.). Second, it should be simple, both for

114 the subject to perform and for the experimenter to run and analyse. It should require no complex

115 instructions and depend upon only standard equipment (non-specialist computer

116 hardware/software, audiovisual systems, and simple response devices). Third, it should contain a

117 number of different trial types, which produce activation in different brain networks, in as wide a

118 spatial distribution across the brain as possible. This helps to rule out effects on neurovascular

119 coupling which may differ in spatially remote brain regions. Fourth, it should be general-

120 purpose; applicable to a wide range of different pharmacological (or other) fMRI studies. Fifth, it

121 should be reliable; it should produce robust results within a single-session, and produce reliable

122 results across multiple sessions. This last point is of particular importance, as use of an unreliable

123 control task would constitute an additional confound, however no previous pharmacological

124 fMRI study has explicitly assessed the reliability of its control task. Indeed reliability is

125 relatively seldom formally assessed in fMRI studies (Plichta et al., 2012).

126 Our aim was to develop some basic open-source task paradigms that have been formally

127 evaluated in terms of reliability, for future use in pharmacological studies, or any investigation

128 where simple test paradigms are required, and reliability is an important consideration (e.g.

129 multisite fMRI studies; Brown et al, 2011). We have developed two variants of a task paradigm

130 that meet the above mentioned criteria, and are furthermore programmed in an open-source

131 software environment (PsychoPy; Peirce, 2007, 2008). One variant consists of visual, auditory,

132 motor, and eye-movement trials. The other substitutes a brief working-memory task for the eye-

133 movement trials, but is otherwise identical. Both are short (5 minutes in duration), simple

134 (requiring only standard audiovisual equipment, and a single-button response box), and both

135 produce four robust, distinct, and specific patterns of brain activation in widely-distributed brain

136 regions. The reliability of the task variants across two scanning sessions has been explicitly

137 assessed using a combination of voxel-wise and Region of Interest (ROI) based approaches. 


\section{Methods}

139 Subjects

14015 healthy subjects ( 6 males, 9 females) from ages 21-48 (mean age $=30)$ were scanned on two

141 separate occasions with the average re-test interval being two weeks. All participants were fully

142 briefed and provided written informed consent. All scans were performed under local institution-

143 approved guidelines for MRI scans on healthy subjects to ensure adherence to ICH-GCP

144 standards.

\section{Task Design and Procedure}

146 The tasks were programmed in PsychoPy (Peirce, 2007, 2008); a free, open-source, cross-

147 platform Python library optimized for experimental design. The task was a randomized event-

148 related design, and consisted of 5 discrete trial types: auditory, visual, motor, cognitive and null

149 trials, each lasting exactly three seconds. A small red, square fixation point was present

150 throughout each task (except in one trial type, as noted below) at the centre of the screen.

151 Auditory trials presented six pure tones for $0.5 \mathrm{~s}$ each, at frequencies of $261.63 \mathrm{~Hz}, 293.66 \mathrm{~Hz}$,

$152329.63 \mathrm{~Hz}, 349.23 \mathrm{~Hz}, 440 \mathrm{~Hz}$, and $493.88 \mathrm{~Hz}$ (corresponding to the musical pitches $\mathrm{C}_{4}, \mathrm{D}_{4}, \mathrm{E}_{4}, \mathrm{~F}_{4}$,

$153 \mathrm{~A}_{4}$, and $\mathrm{B}_{4}$, respectively). The order of the six tones was randomly determined on each trial.

154 Visual trials consisted of a centrally-presented sine-wave grating subtending approximately $10^{\circ}$

155 of visual angle and with a spatial frequency of 1.2 cycles/degree. The grating drifted laterally at a 156 rate of 6 cycles per second, and the direction of drift reversed every $0.5 \mathrm{~s}$. Motor trials consisted

157 of three presentations of a small image of a button, presented just above the centre of the screen,

158 for $1 \mathrm{~s}$ each. This was a cue for subjects to press the response box key, and the button image

159 disappeared after each response was made. The 'cognitive' trial differed in the two variations of

160 the task. In the eye-movement variant, the fixation point moved to six different locations

161 corresponding to the compass locations North-East, East, South-East, North-West, West, and

162 South-West. These points were mapped on a circle with a radius of approximately $8.75^{\circ}$ of visual

163 angle. Each location was maintained for $0.5 \mathrm{~s}$, and all six were presented (in a random order) in

164 each three second trial. In the working-memory variant of the experiment, the cognitive trial

165 consisted of a brief working memory task. This involved the presentation of two letter strings

166 (containing four letters each), followed by a single letter. The subject's task was to indicate

167 whether the final, single letter was present in the first letter string. If the final letter was present 
168 in the first letter string, they were instructed to push the response button. If the final letter was

169 not present in the first letter string they were instructed to make no response. For half the

170 working memory trials the final letter was present in the first string, and for half it was not

171 present. Finally, in the null trials the fixation point was maintained for three seconds, with no

172 other stimuli presented.

173 The two task variants were identical, except for the inclusion of eye-movement trials in one, and

174 working-memory trials in the other. Each task consisted of 100 trials (20 of each of the four

175 active conditions, plus 20 null trials) presented in a standardized pseudo-random order. Separate

176 versions of the two tasks reversed the trial order, and the order of presentation of these versions

177 was counter-balanced across subjects and scans. The order of presentation of the two task

178 variants in the scan sessions was also systematically varied across subjects and scans. The task

179 durations were exactly five minutes (100 trials of $3 \mathrm{~s}$ duration) plus a 10 second buffer period at

180 the end.

181 Prior to each scan session, subjects were shown a short demonstration version of each variant of

182 the task, and instructed on how to perform them. Written instructions were also presented to the

183 subjects in the scanner, immediately prior to the start of each task (see supplementary material

184 for details). During the scanning session, visual stimuli were projected through a wave guide in

185 the rear wall of the scanner room onto a screen mounted in the rear of the scanner bore. This was

186 viewed in a mirror mounted to the head coil. Participants received auditory stimuli via MRI-

187 compatible headphones, and responded using a one-button response box held in their right hand.

188 Responses were recorded using PsychoPy's data-logging routines.

189 All the tasks' PsychoPy code and associated files are available on FigShare at

190 https://figshare.com/articles/fMRI_control_task_zip/5162065 (DOI:

191 10.6084/m9.figshare.5162065; short-link: goo.gl/DAqn4V), and on GitHub at:

192 https://github.com/mattwall1103/fMRI-Control-Task (short-link: https://goo.gl/PcRurT).

193 MRI data acquisition and analysis

194 Data were acquired on a Siemens 3T Magnetom Trio MRI scanner (Siemens Healthcare,

195 Erlangen, Germany), equipped with a 32-channel phased-array head coil. A high-resolution T1-

196 weighted image was acquired at the beginning of each scan using a magnetization prepared rapid 
197 gradient echo (MPRAGE) sequence with parameters from the Alzheimer's Disease Research 198 Network (ADNI; 160 slices x 240 x 256, TR $=2300 \mathrm{~ms}$, TE $=2.98 \mathrm{~ms}$, flip angle $=9^{\circ}, 1 \mathrm{~mm}$ 199 isotropic voxels, bandwidth $=240 \mathrm{~Hz} /$ pixel, parallel imaging factor $=2$; Jack et al, 2008).

200 Functional data collection used an echo-planar imaging (EPI) sequence for BOLD contrast with 20136 axial slices, aligned with the AC-PC axis $\left(\mathrm{TR}=2000 \mathrm{~ms}, \mathrm{TE}=31 \mathrm{~ms}\right.$, flip angle $=80^{\circ}, 3 \mathrm{~mm}$ 202 isotropic voxels, parallel imaging factor $=2$, bandwidth $=2298 \mathrm{~Hz} /$ pixel). Each functional scan 203 lasted five minutes and ten seconds and consisted of 155 volumes.

204 Analysis was completed with FSL version 5.0.4 (FMRIB's software Library; Oxford Centre for 205 Functional Magnetic Resonance Imaging of the Brain; www.fmrib.ox.ac.uk/fs1/). Anatomical 206 Images were initially skull-stripped using BET (Brain Extraction Tool; included in FSL). Images 207 were pre-processed with standard parameters (head-motion correction, $100 \mathrm{~s}$ temporal filtering, 6 $208 \mathrm{~mm}$ spatial smoothing, co-registration to a standard template; MNI152). First-level analysis used 209 a General Linear Model (GLM) approach with the four active conditions modelled as separate 210 (event-series) regressors and the null trials implicitly modelled as the baseline. Also included 211 were the first temporal derivatives of each time-series and an extended set of (24) head-motion 212 parameters as regressors of no interest. Group level analyses computed a simple mean across all 213 subjects within each scan session separately using FSL's FLAME-1 model and a statistical 214 threshold of $Z=3.1, p<0.05$ (cluster-corrected). Contrasts were defined to isolate the response to 215 each trial type relative to the null trials (baseline sections of the time-series). An additional 216 analysis used mid-level fixed effects models to average each subject's responses across the two 217 scan sessions, and then higher-level group analyses (with the same model and thresholds 218 described above) to combine these data across subjects. These were conducted in order to 219 visualize the overall effect of the tasks across subjects, and the two scans. Two separate sets of 220 analyses were conducted, for data from the two task variants.

221 Additional analyses used Intra-Class Correlation (ICC; Shrout \& Fleiss, 1979) coefficients to 222 assess the reliability of responses across the two scanning sessions. This was performed in two 223 ways; using an ROI-based approach, and by generating statistical maps of ICC values in a voxel224 wise manner. For the ROI analysis, five regions were defined based on expected locations of 225 brain activation in the tasks: primary auditory cortex in the superior temporal lobe (bilateral; 226 auditory trials), primary visual cortex in the calcarine sulcus (bilateral; visual trials), left- 
227 hemisphere motor cortex (motor trials), the Frontal Eye Fields (FEF; bilateral; eye-movement 228 trials), and the Dorso-Lateral Pre-Frontal Cortex (DLPFC; bilateral; working memory trials).

229 ROIs were defined as $5 \mathrm{~mm}$-radius spheres, and positioning coordinates were determined using 230 guidance from relevant meta-analytic terms on Neurosynth (http://neurosynth.org/). The ROI

231 definition was therefore performed completely independently from the main experimental data.

232 Activation amplitude data was extracted from these ROIs for all subjects/scans and ICC $(3,1)$

233 statistics were calculated using SPSS (IBM Corp; Armonk, NY).

234 The ICC statistical maps were produced using custom Python code and produced voxelwise 235 images of $\operatorname{ICC}(3,1)$ statistics. For the purposes of thresholding the results, the ICC values were 236 then transformed into standardized values ( $Z$ scores) using the method of Fisher (1915). These 237 images were then thresholded using the same statistical criterion used for the group level BOLD 238 activation analyses; $Z>3.1, p<0.05$ (cluster-corrected for multiple comparisons). These 239 thresholded images were then used to mask the original ICC voxelwise images, to finally 240 produce a robustly thresholded image, which also retains the original, more intuitive, ICC values. 241 Several summary measures were extracted from these images. Firstly, the median ICC values in 242 the thresholded images were calculated for each contrast/task condition. Secondly an analysis 243 was performed in order to assess the relationship between the strength of activation, and the 244 reliability of activation. Following the example of Caceres et al. (2009) median ICC values were 245 calculated using images masks defined by different thresholds on the activation data. The 246 activation data were thresholded at progressively higher $Z$ values (2.3, 3.1, 3.7, and 4.3; 247 equivalent to $\mathrm{p}=0.01, \mathrm{p}=0.001, \mathrm{p}=0.0001$, and $\mathrm{p}=0.00001$, respectively) and these image 248 masks were used to calculate the median ICC scores using the unthresholded ICC images. In 249 order to further examine the spatial relationship between the BOLD activation maps, and the 250 reliability coefficient maps measures of the overlap (percentage of above-threshold voxels in the 251 ICC maps, that were also above-threshold in the activation maps) were also calculated. 


\section{Results}

253 Behavioural performance

254 Subjects' behaviour was recorded and analysed to verify compliance with the task demands. An 255 average accuracy rate of $93 \%$ was achieved within the working memory task. 94\% and 97\%

256 accuracy was achieved for the motor task within the eye movement variant and working memory

257 variant, respectively. All subjects performed the tasks satisfactorily.

\section{Group-level task activation}

259 All tasks performed as expected and produced robust patterns of brain activity in regions

260 previously shown to be activated by similar tasks. Performance of auditory, visual, and motor

261 components of the tasks was consistent across both task variants (see Figures 1a and 2a).

262 Auditory trials produced strong bilateral activation within the superior temporal regions,

263 consistent with primary auditory cortex (Robson, Dorosz \& Gore, 1998). Visual trials produced

264 activity in posterior calcarine sulcus and the occipital pole (primary visual cortex), and in the

265 lateral visual region V5/MT+ (Smith et al., 2006; Wall et al., 2008). Motor trials produced

266 activity in the left-hemisphere post-central sulcus, consistent with the known location of the hand

267 representation in primary motor cortex (Lotze et al., 2000).

268 In the eye-movement variant of the experiment, the eye-movement task produced activation in 269 the Frontal Eye Fields (FEF), alongside activity within V5/MT+, the anterior portion of the 270 calcarine sulcus/primary visual regions, and the intraparietal sulcus (see Figure 1). This is 271 generally consistent with previous reports of brain activity associated with eye-movement tasks.

272 In the working-memory variant of the experiment, the working memory trials produced a highly

273 robust activation pattern corresponding closely to that shown in conventional working memory

274 tasks, such as the N-back (Owen et al., 2005). These regions included bilateral DLPFC,

275 intraparietal sulcus, superior parietal lobule, dorsal anterior cingulate and the temporo-parietal 276 junction (see Figure 2). Insert Figure 1 here.

Figure 1. Results from the eye-movement variant of the task paradigm. Results of grouplevel analyses represented on a cortical surface rendering of a standard anatomical image 
281

282

283

284

285

286

287

288

289

290

291

292

293

294

295

296

297

298

299

300

301

302

303

304

305

306

307

308

309

310

(MNI152). A: Active brain regions for each contrast (mean of both scanning sessions) with functional maps thresholded at $Z>3.1, p<0.05$ (cluster-corrected). B: Results of the reliability analysis comparing session 1 to session 2 ; Intra-class correlation $(3,1)$

maps, masked with a $Z$-transformed, thresholded $(Z>3.1, p<0.05$; cluster-corrected) version in order to produce a robustly-thresholded image, while retaining the original ICC values (see methods for full details). Rows 1-4 are auditory, motor, visual and eyemovement trials. See supplementary tables 1 and 3 for cluster coordinates for all the statistical maps in this figure.

Insert Figure 2 here.

Figure 2. Results from the working-memory variant of the task paradigm. Results of group-level analyses represented on a cortical surface rendering of a standard anatomical image (MNI152). A: Active brain regions for each contrast (mean of both scanning sessions) with functional maps thresholded at $Z>3.1, p<0.05$ (cluster-corrected). B:

Results of the reliability analysis comparing session 1 to session 2; Intra-class correlation $(3,1)$ maps, masked with a $Z$-transformed, thresholded $(Z>3.1, p<0.05$; clustercorrected) version in order to produce a robustly-thresholded image, while retaining the original ICC values (see methods for full details). Rows 1-4 are auditory, motor, visual and working memory trials. See supplementary tables 2 and 4 for cluster coordinates for all the statistical maps in this figure.

Parameter estimate data were extracted from each contrast using a set of five ROIs: primary auditory cortex (auditory trials), frontal eye-fields (eye-movement trials), left-hemisphere primary motor cortex (motor trials), primary visual cortex (visual trials), and dorsolateralprefrontal cortex (working memory trials). These data are plotted for each condition and scan session in Figure 3. Statistical analysis of these data used paired $t$-tests to compare data from each contrast across the two scanning sessions, and a Bonferroni-corrected alpha value of $p<$ 0.00625 (corrected for 8 comparisons). None of the comparisons showed significant results.

Insert Figure 3 here.

Figure 3. ROI data for each task condition within the two task variants $(\mathrm{A}=$ eye- 
movement variant, $B=$ working-memory variant). Units are parameter estimates resulting

312

313

314

315

316

317

318

319

320

321

322

323

324

325

326

327

328

329

330

331

332

333

334

335

336

337

338 from each of the four contrasts in each GLM analysis, relative to baseline (null trials) and are arbitrary units. ROIs are primary auditory cortex (auditory trials; green), lefthemisphere primary motor cortex (motor trials; magenta), primary visual cortex (visual trials; blue), the frontal eye-fields (eye-movement trials; red; panel A), and dorsolateralprefrontal cortex (working memory trials; red; panel B). Grey histogram bars depict the mean of each condition. See supplementary Figure 3 for images of the ROIs.

\section{Reliability analyses}

To assess voxel level reliability, intra-class correlation $(\operatorname{ICC}(3,1))$ maps were created for each task (Figures 1 and 2; right columns). These show a spatial distribution similar to the activation maps, with peak reliability estimates generally corresponding to the location of peak task-related activation. Reliability estimates in the working-memory variant of the task were generally higher and more widespread than in the eye-movement variant. The median ICC value in the thresholded maps is greater than 0.7 in all cases (see tables 1 and 2 below). For additional visualizations of the spatial correspondence between the activation maps and the ICC results, see supplementary Figures 1 and 2.

In the ROI analysis, one task condition (working memory) had a value $>0.75$ which is classed as 'excellent' under Cicchetti's (1994) scheme for interpretation of ICC results. Three ROIs/conditions featured ICC values of 0.6 or above, which is classed as 'good'. A further three ROIs had values in the range 0.4-0.59 which is classed as 'fair' reliability. Only one (visual trials, in the eye-movement variant) was $<0.4$, and thus classed as 'poor'. The working-memory variant generally showed more consistent and higher reliability values than the eye-movement variant, in this analysis.

In the analysis of ICC values at different activation thresholds, the activation data were progressively thresholded at higher levels, and these image masks used to produce median ICC values from the (unthresholded) ICC maps. Reliability measures from this procedure (see Tables 1 and 2) were generally poor, with only one task condition (auditory trials in the eye-movement 
339 variant) $>0.6$ (i.e. 'good' reliability) and many other values $<0.4$ (i.e. 'poor' reliability).

340 Unthresholded statistical maps resulting from all the group-level analyses (brain activation maps, 341 and the voxel-wise ICC maps) are available to view at: https://neurovault.org/collections/3264/.

342 All raw data from this study is also available at:

343 https://openneuro.org/datasets/ds001344/versions/00001 (DOI:

344 10.18112/openneuro.ds001344.v1). 
345

346

347

348

349

350

351

352

353

354

355

356

357

358

359

360

361

362

363

364

365
Insert Table 1 here.

Table 1. ICC $(3,1)$ values for the eye-movement variant of the task. The first column shows the median ICC values in the (thresholded) ICC $(3,1)$ maps, for each task condition. The second column shows $\operatorname{ICC}(3,1)$ calculated from BOLD amplitude data in pre-defined ROIs. The next four columns show median $\operatorname{ICC}(3,1)$ values from the unthresholded ICC maps, within image masks produced by thresholding the taskactivation maps at progressively more conservative levels $(Z=2.3$, to $Z=4.3)$. The final column shows the percentage of voxels in the (thresholded) $\operatorname{ICC}(3,1)$ maps that are also above-threshold in the task activation maps.

Insert Table 2 here.

Table 2. ICC $(3,1)$ values for the working memory variant of the task. The first column shows the median ICC values in the (thresholded) ICC $(3,1)$ maps, for each task condition. The second column shows $\operatorname{ICC}(3,1)$ calculated from BOLD amplitude data in pre-defined ROIs. The next four columns show median $\operatorname{ICC}(3,1)$ values from the unthresholded ICC maps, within image masks produced by thresholding the taskactivation maps at progressively more conservative levels $(Z=2.3$, to $Z=4.3)$. The final column shows the percentage of voxels in the (thresholded) $\operatorname{ICC}(3,1)$ maps that are also above-threshold in the task activation maps. 


\section{Discussion}

367 We have developed and successfully validated two variants of a novel fMRI control task and 368 demonstrated that they show good test-retest reliability. These tasks are short (five minutes 369 duration), relatively simple for both the experimenter and subject (they require only standard 370 audio-visual presentation equipment and a one-button response box), are highly robust in terms

371 of the amplitude of brain activation produced, and show strong reliability features across two 372 sessions. Each variant also produces a number of useful readouts (visual, auditory, motor, 373 cognitive/eye-movements) in a wide spatial distribution across the brain.

374 Both task variants performed similarly for visual, auditory, and motor trials, with robust activity 375 seen in primary visual, auditory, and motor cortex respectively, and little 'off-target' activation evident. The eye-movement task also produced a characteristic pattern of brain activity similar to

377 that seen in previous eye-movement studies (e.g. Berman et al., 1999). The working memory 378 task, though only requiring a very brief (two-second) retention interval, produced a highly 379 similar pattern of activity to that seen in more standard working memory tasks such as the N380 back task (Owen et al., 2005).

381 Importantly, reliability of the tasks was also assessed, and found to be generally high. Reliability 382 assessment using ICC (or other measures) is still relatively uncommon for fMRI experiments, 383 but is an important step in validating task paradigms (Caceres et al., 2009). The ICC measures 384 obtained here compare favourably with previous reports using auditory and working memory 385 tasks (Caceres et al., 2009), a cognitive-emotive test battery (Plichta et al., 2012), and a reward 386 task (Fliessbach et al., 2010). However, some task conditions were seen to be more reliable than 387 others. In particular, reliability in the working-memory variant of the experiment was generally 388 higher than in the eye-movement variant. One possible explanation for this difference may be due to the much more cognitively demanding features of the working-memory variant, which led to a higher level of attention and engagement to all the task conditions in that variant.

391 Previous work by Caceres et al. (2009) showed that the median ICC value tended to increase in 392 regions defined by progressively higher activation thresholds; suggesting an association between 393 higher BOLD activation, and higher reliability. This seems not to be the case in the current data, 394 with profiles of ICC values remaining flat at different thresholds (see Tables 1 and 2, and 
395 supplementary Figure 7), with relatively poor (though broadly comparable to Caceres et al., 396 2009) ICC coefficients, when derived in this way. Quantification of the spatial concordance 397 between the activation and ICC maps showed that the sensory (auditory and visual) conditions 398 share a much higher proportion of voxels than the other (motor, cognitive) conditions, with rates 399 of $<10 \%$ for the latter in some cases. However, the median ICC values in these conditions are 400 still high $(>0.7)$. This suggests that there may be a distinction between brain regions which are 401 robustly activated by a task, and brain regions which are reliably modulated by a task across two 402 sessions, and that the extent of this dissociation also depends on the task/conditions used. The 403 task conditions that produce activation in relatively focused and discrete brain regions (i.e. 404 primary auditory and visual cortices) show higher spatial concordance than those tasks which 405 activate much broader brain networks. As noted above, there is a paucity of studies that have 406 explicitly assessed the test-retest reliability of fMRI, so much more work with different task 407 paradigms and approaches will be required to further investigate these findings.

408 The high reliability, short duration, and ease of use of these tasks make them ideal for inclusion 409 as control tasks in pharmacological-MRI studies, as suggested by Iannetti and Wise (2007), and 410 Bourke and Wall (2015). Inclusion of tasks which are (hypothetically) unaffected by the drug 411 helps rule out alternative explanations related to systemic drug effects (on blood pressure, heart412 rate, etc.), or neuro-vascular coupling, which can both theoretically modulate the BOLD 413 response. One previous study investigating modulation of amygdala responses by citalopram 414 (Murphy et al., 2009) used a simple checker-board visual control task. Use of a single control 415 task where activation is restricted to the occipital lobe is sub-optimal as the drug may potentially 416 still produce non-neural effects in other brain regions. A recent study on the brain effects of the 417 sex hormone kisspeptin (Comninos et al., 2017) used a control task with a number of readouts in 418 different brain regions (based on Pinel et al., 2007). This task was complex, with ten individual 419 stimulus conditions, different response options, and contained high-level cognitive stimuli 420 (performing mental arithmetic, reading sentences on the screen, and listening to recorded voices) 421 which included culture- and language-specific features. This complexity and the use of language422 specific stimuli limit the broad applicability of this task.

423 Use of control tasks in this manner can bolster support for a particular interpretation of a study's 424 outcomes, however they may never be conclusive. A successful outcome of these tasks (when 
425 used in the context of a pharmacological study) would be a lack of effect of the drug treatment, 426 and strong inference from a null effect is an issue for standard statistical approaches based on 427 null-hypothesis significance testing (Neyman \& Pearson, 1933). Ideally, the experiment would 428 be well-powered to detect such an effect, however recent meta-analytic work has demonstrated 429 that low experimental power is an endemic problem in neuroscience research (Button et al., 430 2013, although see Nord et al., 2017, for a somewhat more optimistic assessment). Use of 431 Bayesian statistics is an alternative approach that may mitigate this issue and can provide a 432 coherent method for determining whether a null result is 'genuine' or simply resulting from 433 insensitivity of the data (Dienes, 2014, 2016).

434 The tasks evaluated here represent a good compromise between ease of use, wide applicability, a 435 short duration, reliable results, and the desirability of providing a number of readouts in spatially 436 diverse brain regions. While the working memory variant appears to be somewhat more robust, 437 more reliable, and produces a wider pattern of brain activity, it is also more cognitively 438 demanding and has significantly more complex instructions. This may make it less suitable for 439 any patient group with significant cognitive impairments, who may struggle with a fast, 440 demanding task. The eye-movement variant may therefore be more suitable for these groups. 441 Additionally, the eye-movement variant may also be more suitable where the drug under 442 investigation is hypothesized to have an effect on cognition. In this case, the working-memory 443 variant may be inappropriate as a control task, as it strongly engages well-known cognitive brain 444 regions. Either variant would also be suitable for use in a number of other situations where a 445 short, reliable fMRI task that yields a number of readouts is required, for example in systematic 446 testing of fMRI acquisition sequence parameters (as in Demetriou et al., 2018). Standardization 447 of task paradigms, data acquisition practices, and analysis procedures in fMRI is currently at a 448 nascent stage. Encouraging progress is being made on some aspects (e.g. Esteban et al., 2018) 449 however the huge variety of experimental designs and analysis strategies in current use still 450 impedes direct comparison of studies. Open-source tasks that have undergone some kind of 451 formal evaluation or validation (such as those presented here) could therefore be a useful tool in 452 future efforts at standardization. We have evaluated two variants of a novel task paradigm, 453 suitable for use as a control task in pharmacological fMRI studies, or for any use where a 454 general-purpose battery of basic tasks/stimuli is required. The tasks produce robust brain 455 activation and have strongly favourable reliability features. The tasks are programmed in an 
456 open-source language and experimental presentation application (Python/PsychoPy), and we 457 have therefore made the stimulus code freely available on FigShare at 458 https://figshare.com/articles/fMRI_control_task_zip/5162065 (DOI:

459 10.6084/m9.figshare.5162065; short-link: goo.gl/DAqn4V), and on GitHub at:

460 https://github.com/mattwall1103/fMRI-Control-Task (short-link: https://goo.gl/PcRurT). We

461 encourage any interested researchers to download the programs and use them in their research. 


\section{References}

463

464

465

466

467

468

469

470

471

472

473

474

475

476

477

478

479

480

481

482

483

484

485

486

487

488

489

490

491

492

493

494

495

496

497

498

499

500

501

502

503

504

505

506
Baillet S. 2017. Magnetoencephalography for brain electrophysiology and imaging. Nature Neuroscience 20:327-339. DOI: 10.1038/nn.4504.

Berman RA., Colby C., Genovese C., Voyvodic J., Luna B., Thulborn K., Sweeney J. 1999. Cortical networks subserving pursuit and saccadic eye movements in humans: an FMRI study. Human Brain Mapping 8:209-225.

Bourke JH., Wall MB. 2015. phMRI: methodological considerations for mitigating potential confounding factors. Frontiers in Neuroscience 9:1-7. DOI: 10.3389/fnins.2015.00167.

Brown GG., Mathalon DH., Stern H., Ford J., Mueller B., Greve DN., McCarthy G., Voyvodic J., Glover G., Diaz M., Yetter E., Ozyurt IB., Jorgensen KW., Wible CG., Turner JA., Thompson WK., Potkin SG. 2011. Multisite reliability of cognitive BOLD data. NeuroImage 54:2163-2175. DOI: 10.1016/j.neuroimage.2010.09.076.

Buxton RB., Wong EC., Frank LR. 1998. Dynamics of Blood Flow and Oxygenation Changes During Brain Activation : The Balloon Model. Magnetic resonance in medicine 39:855864.

Caceres A., Hall DL., Zelaya FO., Williams SCR., Mehta MA. 2009. Measuring fMRI reliability with the intra-class correlation coefficient. NeuroImage 45:758-768. DOI: 10.1016/j.neuroimage.2008.12.035.

Carhart-Harris RL., Wall MB., Erritzoe D., Kaelen M., Ferguson B., De Meer I., Tanner M., Bloomfield M., Williams TM., Bolstridge M., Stewart L., Morgan CJ., Newbould RD., Feilding A., Curran H V., Nutt DJ. 2014. The effect of acutely administered MDMA on subjective and BOLD-fMRI responses to favourite and worst autobiographical memories. Int J Neuropsychopharmacol 17:527-540. DOI: 10.1017/S1461145713001405.

Carmichael O., Schwarz AJ., Chatham CH., Scott D., Turner JA., Upadhyay J., Coimbra A., Goodman JA., Baumgartner R., English BA., Apolzan JW., Shankapal P., Hawkins KR. 2018. The role of fMRI in drug development. Drug Discovery Today 23:333-348. DOI: 10.1016/j.drudis.2017.11.012.

Cicchetti D V. 1994. Guidelines, criteria, and rules of thumb for evaluating normed and standardized assessment instruments in psychology. Psychological Assessment 6:284-290. DOI: $10.1037 / 1040-3590.6 .4 .284$.

Comninos AN., Wall MB., Demetriou L., Shah AJ., Clarke SA., Narayanaswamy S., Nesbitt A., Izzi-engbeaya C., Prague JK., Abbara A., Ratnasabapathy R., Salem V., Nijher GM., Jayasena CN., Tanner M., Bassett P., Mehta A., Rabiner EA., Hönigsperger C., Silva MR., Brandtzaeg OK., Lundanes E., Wilson SR., Brown RC., Thomas SA., Bloom SR., Dhillo WS. 2017. Kisspeptin modulates sexual and emotional brain processing in humans. Journal of Clinical Investigation 127:709-719.

Demetriou L., Kowalczyk OS., Tyson G., Bello T., Newbould RD., Wall MB. 2018. A comprehensive evaluation of increasing temporal resolution with multiband-accelerated sequences and their effects on statistical outcome measures in fMRI. NeuroImage 176:404416.

Dienes Z. 2014. Using Bayes to get the most out of non-significant results. Frontiers in Psychology 5:1-17. DOI: 10.3389/fpsyg.2014.00781.

Dienes Z. 2016. How Bayes factors change scientific practice. Journal of Mathematical Psychology 72:78-89. DOI: 10.1016/j.jmp.2015.10.003.

Diukova A., Ware J., Smith JE., Evans CJ., Murphy K., Rogers PJ., Wise RG. 2012. Separating 
507

508

509

510

511

512

513

514

515

516

517

518

519

520

521

522

523

524

525

526

527

528

529

530

531

532

533

534

535

536

537

538

539

540

541

542

543

544

545

546

547

548

549

550

551

552

neural and vascular effects of caffeine using simultaneous EEG-FMRI: differential effects of caffeine on cognitive and sensorimotor brain responses. NeuroImage 62:239-49. DOI: 10.1016/j.neuroimage.2012.04.041.

Esteban O., Markiewicz C., Blair RW., Moodie C., Isik AI., Aliaga AE., Kent J., Goncalves M., DuPre E., Snyder M., Oya H., Ghosh S., Wright J., Durnez J., Poldrack R., Gorgolewski KJ. 2018. FMRIPrep: a robust preprocessing pipeline for functional MRI. bioRxiv:306951. DOI: $10.1101 / 306951$.

Fisher RA. 1915. Frequency Distribution of the Values of the Correlation Coefficient in Samples from an Indefinitely Large Population. Biometrika 10:507. DOI: 10.2307/2331838.

Fliessbach K., Rohe T., Linder NS., Trautner P., Elger CE., Weber B. 2010. Retest reliability of reward-related BOLD signals. NeuroImage 50:1168-76. DOI:

10.1016/j.neuroimage.2010.01.036.

Friston KJ., Mechelli a., Turner R., Price CJ. 2000. Nonlinear responses in fMRI: the Balloon model, Volterra kernels, and other hemodynamics. NeuroImage 12:466-77. DOI:

10.1006/nimg.2000.0630.

Iannetti GD., Wise RG. 2007. BOLD functional MRI in disease and pharmacological studies: room for improvement? Magnetic resonance imaging 25:978-88. DOI: 10.1016/j.mri.2007.03.018.

Jack CR., Bernstein MA., Fox NC., Thompson P., Alexander G., Harvey D., Borowski B., Britson PJ., Whitwell JL., Ward C., Dale AM., Felmlee JP., Gunter JL., Hill DLG., Killiany R., Schuff N., Fox-Bosetti S., Lin C., Studholme C., DeCarli CS., Krueger G., Ward HA., Metzger GJ., Scott KT., Mallozzi R., Blezek D., Levy J., Debbins JP., Fleisher AS., Albert M., Green R., Bartzokis G., Glover G., Mugler J., Weiner MW. 2008. The Alzheimer's Disease Neuroimaging Initiative (ADNI): MRI methods. Journal of Magnetic Resonance Imaging 27:685-691. DOI: 10.1002/jmri.21049.

Kaelen M., Roseman L., Kahan J., Santos-Ribeiro A., Orban C., Lorenz R., Barrett FS., Bolstridge M., Williams T., Williams L., Wall MB., Feilding A., Muthukumaraswamy S., Nutt DJ., Carhart-Harris R. 2016. LSD modulates music-induced imagery via changes in parahippocampal connectivity. European Neuropsychopharmacology. DOI: 10.1016/j.euroneuro.2016.03.018.

Logothetis NK. 2008. What we can do and what we cannot do with fMRI. Nature 453:869-878. DOI: 10.1038/nature06976.

Logothetis NK., Pauls J., Augath M., Trinath T., Oeltermann A. 2001. Neurophysiological investigation of the basis of the fMRI signal. Nature 412:150-157.

Lotze M., Erb M., Flor H., Huelsmann E., Godde B., Grodd W. 2000. fMRI evaluation of somatotopic representation in human primary motor cortex. NeuroImage 11:473-481. DOI: 10.1006/nimg.2000.0556.

Maron E., Wall M., Norbury R., Godlewska B., Terbeck S., Cowen P., Matthews P., Nutt DJ. 2016. Effect of short-term escitalopram treatment on neural activation during emotional processing. Journal of Psychopharmacology 30:33-39. DOI: 10.1177/0269881115620462.

Matthews P., Rabiner I., Gunn R. 2011. Non-invasive imaging in experimental medicine for drug development. Current Opinion in Pharmacology 11:501-7. DOI: 10.1016/j.coph.2011.04.009.

Murphy SE., Norbury R., O’Sullivan U., Cowen PJ., Harmer CJ. 2009. Effect of a single dose of citalopram on amygdala response to emotional faces. The British journal of psychiatry: the journal of mental science 194:535-40. DOI: 10.1192/bjp.bp.108.056093. 
553 Neyman J., Pearson ES. 1933. On the Problem of the Most Efficient Tests of Statistical

554

555

556

557

558

559

560

561

562

563

564

565

566

567

568

569

570

571

572

573

574

575

576

577

578

579

580

581

582

583

584

585

586

587

588

589

590

591

592

593

594

595

596

597

598
Hypotheses. Philosophical Transactions of the Royal Society A: Mathematical, Physical and Engineering Sciences 231:289-337. DOI: 10.1098/rsta.1933.0009.

Nord CL., Valton V., Wood J., Roiser JP. 2017. Power-up: a reanalysis of "power failure" in neuroscience using mixture modelling. The Journal of Neuroscience 37:3592-16. DOI: 10.1523/JNEUROSCI.3592-16.2017.

Owen AM., McMillan KM., Laird AR., Bullmore E. 2005. N-back working memory paradigm: A meta-analysis of normative functional neuroimaging studies. Human Brain Mapping 25:46-59. DOI: $10.1002 / \mathrm{hbm} .20131$.

Peirce J. 2007. PsychoPy— psychophysics software in Python. Journal of neuroscience methods 162:8-13.

Peirce JW. 2008. Generating Stimuli for Neuroscience Using PsychoPy. Frontiers in neuroinformatics 2:10. DOI: 10.3389/neuro.11.010.2008.

Pinel P., Thirion B., Meriaux S. 2007. Fast reproducible identification and large-scale databasing of individual functional cognitive networks. BMC Neuroscience 8:1-18. DOI: 10.1186/1471-2202-8-91.

Plichta MM., Schwarz AJ., Grimm O., Morgen K., Mier D., Haddad L., Gerdes ABM., Sauer C., Tost H., Esslinger C., Colman P., Wilson F., Kirsch P., Meyer-Lindenberg A. 2012. Testretest reliability of evoked BOLD signals from a cognitive-emotive fMRI test battery. NeuroImage 60:1746-58. DOI: 10.1016/j.neuroimage.2012.01.129.

Quelch DR., Mick I., McGonigle J., Ramos AC., Flechais RSA., Bolstridge M., Rabiner E., Wall MB., Newbould RD., Steiniger-Brach B., van den Berg F., Boyce M., Østergaard Nilausen D., Breuning Sluth L., Meulien D., von der Goltz C., Nutt D., Lingford-Hughes A. 2017. Nalmefene Reduces Reward Anticipation in Alcohol Dependence: An Experimental Functional Magnetic Resonance Imaging Study. Biological Psychiatry:1-9. DOI: 10.1016/j.biopsych.2016.12.029.

Robson MD., Dorosz JL., Gore JC. 1998. Measurements of the temporal fMRI response of the human auditory cortex to trains of tones. NeuroImage 7:185-198. DOI: 10.1006/nimg.1998.0322.

Smith A., Wall M., Williams A., Singh K. 2006. Sensitivity to optic flow in human cortical areas MT and MST. European Journal of Neuroscience 23:561-569.

Soares J., Magalhães R., Moreira P., Sousa A., Ganz E., Sampaio A., Alves V., Marques PCG., Sousa N. 2016. A hitchhiker's guide to functional Magnetic Resonance Imaging. Frontiers in Neuroscience 10:515. DOI: 10.3389/FNINS.2016.00515.

Stewart SB., Koller JM., Campbell MC., Black KJ. 2014. Arterial spin labeling versus BOLD in direct challenge and drug-task interaction pharmacological fMRI. PeerJ 2:e687. DOI: 10.7717/peerj.687.

Upadhyay J., Anderson J., Schwarz AJ., Coimbra A., Baumgartner R., Pendse G., George E., Nutile L., Wallin D., Bishop J., Neni S., Maier G., Iyengar S., Evelhoch JL., Bleakman D., Hargreaves R., Becerra L., Borsook D. 2011. Imaging drugs with and without clinical analgesic efficacy. Neuropsychopharmacology 36:2659-73. DOI: 10.1038/npp.2011.156.

Wall MB., Lingnau A., Ashida H., Smith AT. 2008. Selective visual responses to expansion and rotation in the human MT complex revealed by functional magnetic resonance imaging adaptation. The European journal of neuroscience 27:2747-57. DOI: 10.1111/j.14609568.2008.06249.x.

Wise RG., Tracey I. 2006. The role of fMRI in drug discovery. Journal of magnetic resonance 
599 imaging : JMRI 23:862-76. DOI: 10.1002/jmri.20584.

600 Wise RG., Williams P., Tracey I. 2004. Using fMRI to quantify the time dependence of

601

602

603

604

605

606

607

608

609

610 We would like to thank the Imanova Center for Imaging Sciences (Hammersmith Hospital,

611 London, UK, Now Invicro, London) for the scanner time required to complete the project, and

612 general support throughout the investigation 


\section{Figure 1}

Results from the eye-movement variant of the task paradigm.

Results from the eye-movement variant of the task paradigm. Results of group-level analyses represented on a cortical surface rendering of a standard anatomical image (MNI152). A: Active brain regions for each contrast (mean of both scanning sessions) with functional maps thresholded at $Z>3.1, p<0.05$ (cluster-corrected). B: Results of the reliability analysis comparing session 1 to session 2 ; Intra-class correlation $(3,1)$ maps, masked with a $Z$ transformed, thresholded ( $Z>3.1, p<0.05$; cluster-corrected) version in order to produce a robustly-thresholded image, while retaining the original ICC values (see methods for full details). Rows 1-4 are auditory, motor, visual and eye-movement trials. See supplementary tables 1 and 3 for cluster coordinates for all the statistical maps in this figure. 

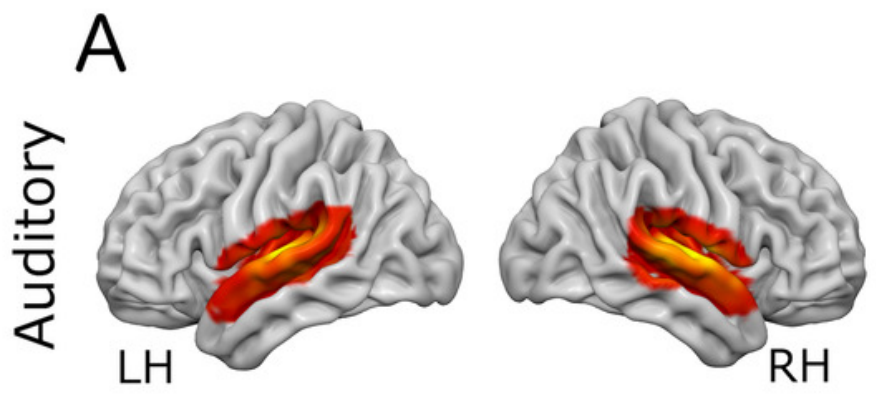

B
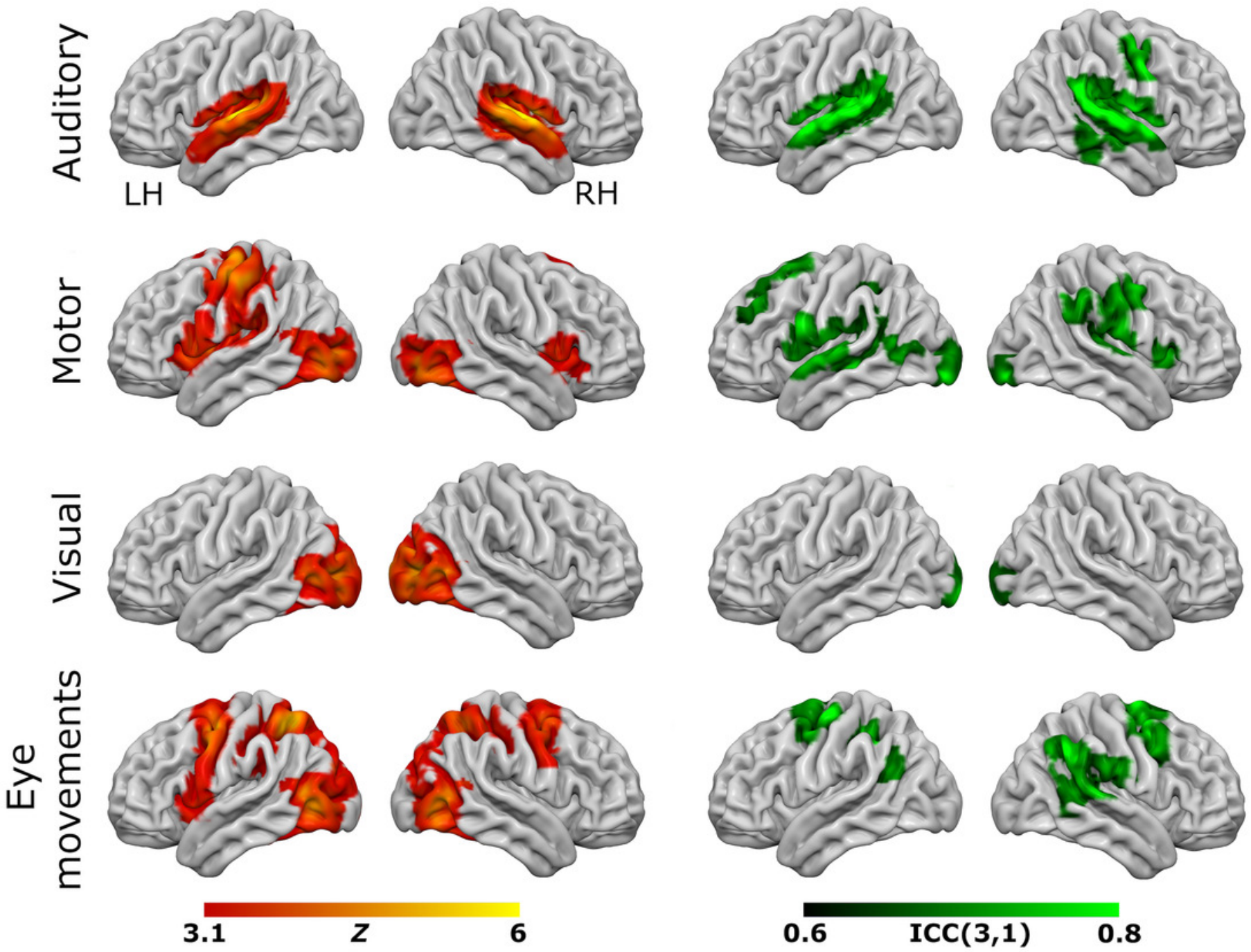


\section{Figure 2}

Results from the working-memory variant of the task paradigm.

Results from the working-memory variant of the task paradigm. Results of group-level analyses represented on a cortical surface rendering of a standard anatomical image (MNI152). A: Active brain regions for each contrast (mean of both scanning sessions) with functional maps thresholded at $Z>3.1, p<0.05$ (cluster-corrected). B: Results of the reliability analysis comparing session 1 to session 2 ; Intra-class correlation $(3,1)$ maps, masked with a $Z$-transformed, thresholded ( $Z>3.1, p<0.05$; cluster-corrected) version in order to produce a robustly-thresholded image, while retaining the original ICC values (see methods for full details). Rows 1-4 are auditory, motor, visual and working memory trials. See supplementary tables 2 and 4 for cluster coordinates for all the statistical maps in this figure. 
A
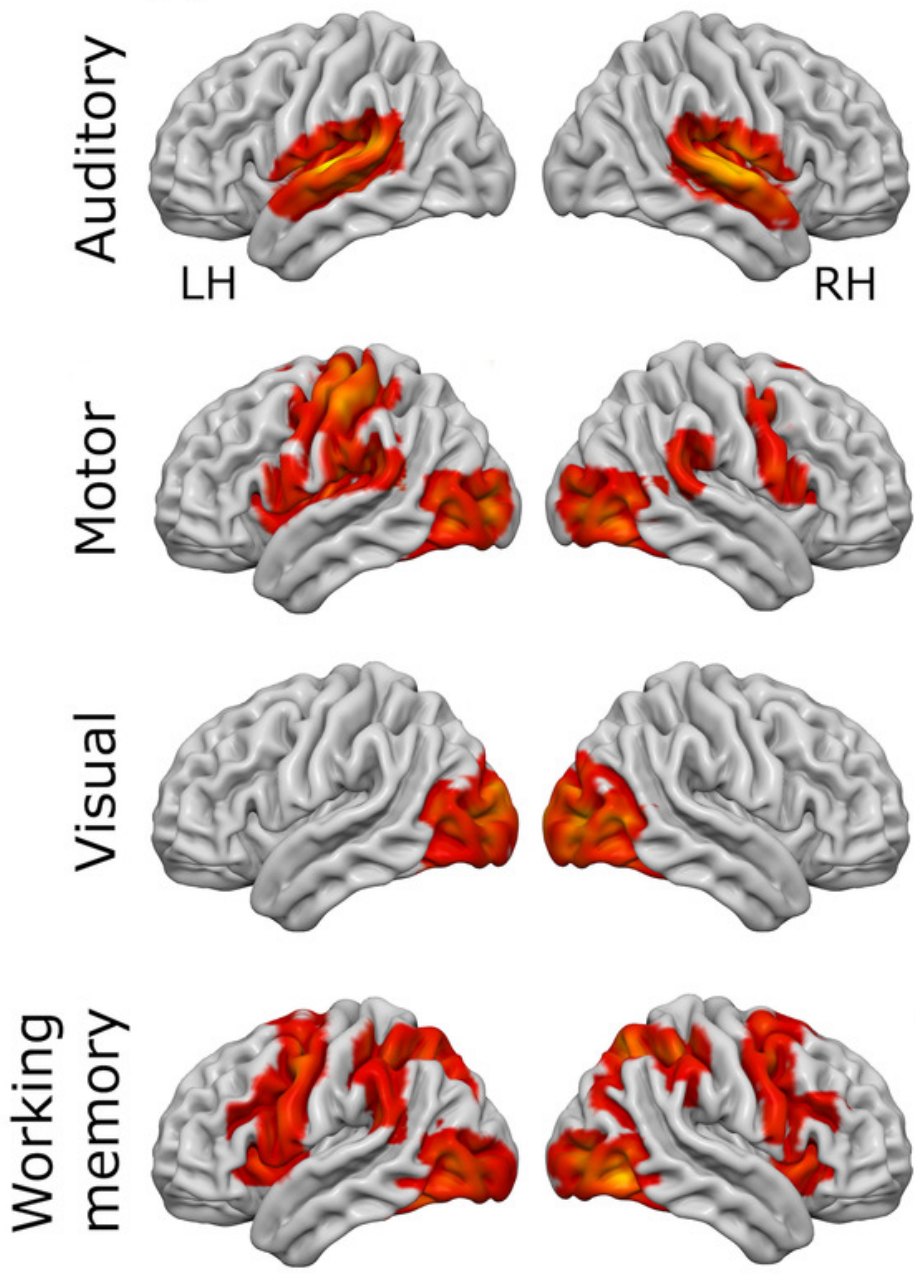
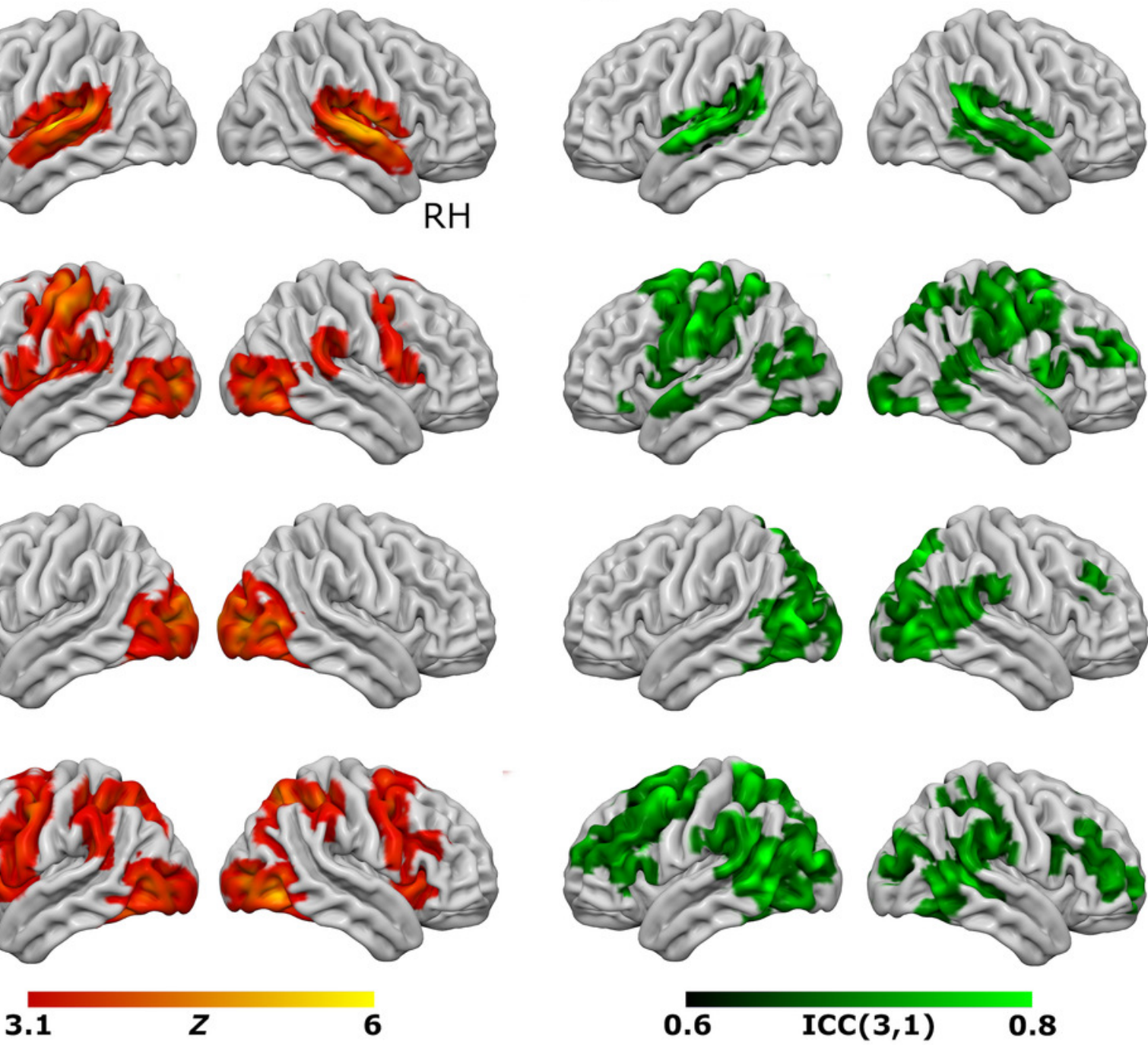
Figure 3

ROI data for each task condition within the two task variants $(A=$ eye-movement variant, $\mathrm{B}=$ working-memory variant).

Units are parameter estimates resulting from each of the four contrasts in each GLM analysis, relative to baseline (null trials) and are arbitrary units. ROls are primary auditory cortex (auditory trials; green), left-hemisphere primary motor cortex (motor trials; magenta), primary visual cortex (visual trials; blue), the frontal eye-fields (eye-movement trials; red; panel A), and dorsolateral-prefrontal cortex (working memory trials; red; panel B). Grey histogram bars depict the mean of each condition. See supplementary Figure 3 for images of the ROls.
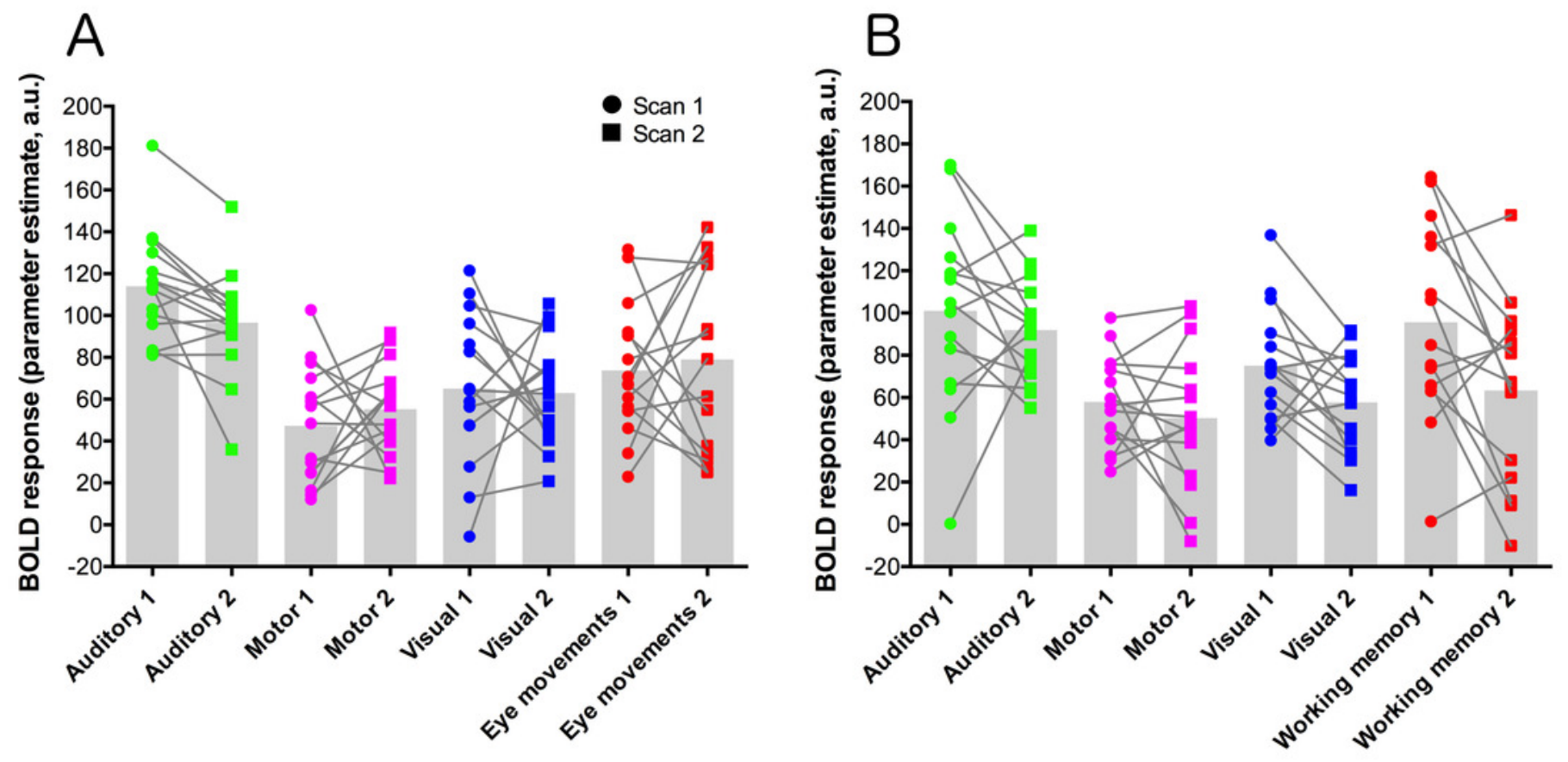


\section{Table $\mathbf{1}$ (on next page)}

$\operatorname{ICC}(3,1)$ values for the eye-movement variant of the task.

The first column shows the median ICC values in the (thresholded) ICC $(3,1)$ maps, for each task condition. The second column shows ICC $(3,1)$ calculated from BOLD amplitude data in pre-defined ROIs. The next four columns show median ICC $(3,1)$ values from the unthresholded ICC maps, within image masks produced by thresholding the task-activation maps at progressively more conservative levels ( $Z=2.3$, to $Z=4.3$ ). The final column shows the percentage of voxels in the (thresholded) ICC $(3,1)$ maps that are also above-threshold in the task activation maps. 
1

\begin{tabular}{|c|c|c|c|c|c|c|c|}
\hline \multirow[t]{2}{*}{$\begin{array}{l}\text { Task } \\
\text { condition }\end{array}$} & \multirow[t]{2}{*}{$\begin{array}{c}\text { Median of } \\
\text { thresholded } \\
\text { ICC }(3,1) \text { maps }\end{array}$} & \multirow[t]{2}{*}{$\begin{array}{l}\operatorname{ICC}(3,1) \\
\text { values (in } \\
\text { ROIs) }\end{array}$} & \multicolumn{4}{|c|}{$\begin{array}{l}\text { Median of ICC values in image } \\
\text { masks produced by thresholding } \\
\text { activation maps }\end{array}$} & \multirow[t]{2}{*}{$\begin{array}{c}\text { Overlap } \\
\text { between ICC } \\
\text { and activation } \\
\text { maps }(\%)\end{array}$} \\
\hline & & & $\mathrm{Z}=\mathbf{2 . 3}$ & $\mathrm{Z}=3.1$ & $\mathrm{Z}=\mathbf{3 . 7}$ & $\mathrm{Z}=4.3$ & \\
\hline Auditory & 0.77 & 0.59 & 0.61 & 0.66 & 0.68 & 0.67 & 66.0 \\
\hline Visual & 0.7 & 0.17 & 0.14 & 0.24 & 0.27 & 0.26 & 55.5 \\
\hline Motor & 0.71 & 0.75 & 0.23 & 0.22 & 0.18 & 0.12 & 7.2 \\
\hline $\begin{array}{l}\text { Eye- } \\
\text { movement }\end{array}$ & 0.71 & 0.58 & 0.21 & 0.22 & 0.21 & 0.15 & 17.6 \\
\hline
\end{tabular}

2 


\section{Table 2 (on next page)}

$\operatorname{ICC}(3,1)$ values for the working memory variant of the task.

The first column shows the median ICC values in the (thresholded) ICC $(3,1)$ maps, for each task condition. The second column shows ICC $(3,1)$ calculated from BOLD amplitude data in pre-defined ROIs. The next four columns show median ICC $(3,1)$ values from the unthresholded ICC maps, within image masks produced by thresholding the task-activation maps at progressively more conservative levels ( $Z=2.3$, to $Z=4.3$ ). The final column shows the percentage of voxels in the (thresholded) ICC $(3,1)$ maps that are also above-threshold in the task activation maps. 
1

\begin{tabular}{|c|c|c|c|c|}
\hline $\begin{array}{l}\text { Task } \\
\text { condition }\end{array}$ & $\begin{array}{c}\text { Median of } \\
\text { thresholded } \\
\text { ICC }(3,1) \text { maps }\end{array}$ & $\begin{array}{l}\operatorname{ICC}(3,1) \\
\text { values (in } \\
\text { ROIs) }\end{array}$ & $\begin{array}{l}\text { Median of ICC values in image } \\
\text { masks produced by thresholding } \\
\text { activation maps }\end{array}$ & $\begin{array}{c}\text { Overlap } \\
\text { between ICC } \\
\text { and activation } \\
\text { maps }(\%)\end{array}$ \\
\hline
\end{tabular}

\begin{tabular}{lccccccc}
\hline & & & $\mathbf{Z}=\mathbf{2 . 3}$ & $\mathbf{Z}=\mathbf{3 . 1}$ & $\mathbf{Z}=\mathbf{3 . 7}$ & $\mathbf{Z}=\mathbf{4 . 3}$ & \\
\hline Auditory & 0.73 & 0.45 & 0.43 & 0.45 & 0.46 & 0.44 & 62.9 \\
Visual & 0.73 & 0.65 & 0.42 & 0.43 & 0.41 & 0.38 & 30.4 \\
Motor & 0.71 & 0.6 & 0.3 & 0.28 & 0.24 & 0.22 & 7.1 \\
$\begin{array}{l}\text { Working } \\
\text { memory }\end{array}$ & 0.72 & 0.76 & 0.4 & 0.39 & 0.35 & 0.23 & 9.5
\end{tabular}

